\title{
ON $g \boldsymbol{W}$-CONTINUOUS FUNCTIONS INDUCED BY GENERALIZED $w$-OPEN SETS IN $w$-SPACES
}

\author{
Won Keun Min \\ Department of Mathematics \\ Kangwon National University \\ Chuncheon, 200-701, KOREA
}

\begin{abstract}
The purpose of this paper is to introduce the notions of $g W$-continuous, $g W^{*}$ continuous, $g W$-irresolute, and $g W^{*}$-irresolute functions induced by $g w$-open sets in $w$-spaces, and to study characterizations and the relationships among them.
\end{abstract}

AMS Subject Classification: 54A05, 54B10, 54C10, 54D30

Key Words: $w$-space, generalized $w$-open, $W$-continuous, $W^{*}$-continuous, $g W$-continuous, $g W^{*}$-continuous, $g W$-irresolute, $g W^{*}$-irresolute

\section{Introduction}

Siwiec [16] introduced the notions of weak neighborhoods and weak base in a topological space. We introduced the weak neighborhood systems defined by using the notion of weak neighborhoods in [11]. The weak neighborhood system induces a weak neighborhood space which is independent of neighborhood spaces [4] and general topological spaces [2]. The notions of weak structure, $w$-space, $W$-continuity and $W^{*}$-continuity were investigated in [12].

Levine [5] introduced the notion of $g$-closed subsets in topological spaces. In fact, the set of all $g$-closed subsets is a kind of weak structure. In the same way, we introduced the notion of generalized $w$-closed set (simply, gw-closed set) [14] in weak spaces, and investigated some basic properties of such notions.

$\begin{array}{lr}\text { Received: } & \text { November 15, } 2016 \\ \text { Revised: } & \text { December 25, } 2016 \\ \text { Published: } & \text { February 1, } 2017\end{array}$

(C) 2017 Academic Publications, Ltd. url: www.acadpubl.eu 
The purpose of this paper is to introduce the notions of $g W$-continuous, $g W^{*}$-continuous, $g W$-irresolute, and $g W^{*}$-irresolute functions induced by $g w$ open sets in $w$-spaces, and to study characterizations and the relationships among such notions, $W$-continuity and $W^{*}$-continuity in $w$-spaces.

\section{Preliminaries}

Let $X$ be a nonempty set. A subfamily $w_{X}$ of the power set $P(X)$ is called a weak structure on $X$ if it satisfies the following:

(1) $\emptyset \in w_{X}$ and $X \in w_{X}$.

(2) For $U_{1}, U_{2} \in w_{X}, U_{1} \cap U_{2} \in w_{X}$.

Then the pair $\left(X, w_{X}\right)$ is called a $w$-space on $X$. Then $V \in w_{X}$ is called a $w$-open set and the complement of a $w$-open set is a $w$-closed set. The collection of all $w$-open sets (resp., $w$-closed sets) in a $w$-space $X$ will be denoted by $W(X)$ (resp., $W C(X))$. We set $W(x)=\{U \in W(X): x \in U\}$.

Let $S$ be a subset of a topological space $X$. The closure (resp., interior) of $S$ will be denoted by $c l S$ (resp., int $S$ ). A subset $S$ of $X$ is called a preopen set [9] (resp., $\alpha$-open set [15], semi-open [6]) if $S \subset \operatorname{int}(\operatorname{cl}(S))$ (resp., $S \subset$ $\operatorname{int}(\operatorname{cl}(\operatorname{int}(S))), S \subset \operatorname{cl}(\operatorname{int}(S)))$. The complement of a preopen set (resp., $\alpha$ open set, semi-open) is called a preclosed set (resp., $\alpha$-closed set, semi-closed). The family of all preopen sets (resp., $\alpha$-open sets, semi-open sets) in $X$ will be denoted by $P O(X)$ (resp., $\alpha(X), S O(X)$ ). We know the family $\alpha(X)$ is a topology finer than the given topology on $X$. And a subset $A$ of $X$ is said to be $g$-closed [5] (resp., gp-closed [7], gs-closed [1,3]) if $\operatorname{cl}(A)$ (resp., $p C l(A)$, $s C l(A)) \subset U$ whenever $A \subset U$ and $U$ is open in $X$.

Then the family $\tau, G O(X), g \alpha O(X)$, and $g \alpha^{*} O(X)$, are all weak structures on $X$. But $P O(X), G P O(X)$ and $S O(X)$ are not weak structures on $X$. A subfamily $m_{X}$ of the power set $P(X)$ of a nonempty set $X$ is called a minimal structure on $X$ [8] if $\emptyset \in w_{X}$ and $X \in w_{X}$. Thus clearly every weak structure is a minimal structure.

For a subset $A$ of $X$, the $w$-closure of $A$ and the $w$-interior of $A$ are defined as follows:

(1) $w C(A)=\cap\left\{F: A \subseteq F, X-F \in w_{X}\right\}$.

(2) $w I(A)=\cup\left\{U: U \subseteq A, U \in w_{X}\right\}$.

Theorem 2.1 ([12]). Let $\left(X, w_{X}\right)$ be a $w$-space and $A \subseteq X$.

(1) $x \in w I(A)$ if and only if there exists an element $U \in W(x)$ such that $U \subseteq A$.

(2) $x \in w C(A)$ if and only if $A \cap V \neq \emptyset$ for all $V \in W(x)$. 
(3) If $A \subseteq B$, then $w I(A) \subseteq w I(B)$; $w C(A) \subset w C(B)$.

(4) $w C(X-A)=X-w I(A) ; w I(X-A)=X-w C(A)$.

(5) If $A$ is $w$-closed (resp., w-open), then $w C(A)=A$ (resp., $w I(A)=A$ ).

Let $\left(X, w_{X}\right)$ be a $w$-space and $A \subseteq X$. Then $A$ is called a generalized $w$ closed set (simply, gw-closed set) [14] if $w C(A) \subseteq U$, whenever $A \subseteq U$ and $U$ is $w$-open. Then the union of two $g w$-closed sets is a $g w$-closed set, but the intersection of two $g w$-closed sets is not always $g w$-closed. The family of all $w$ closed sets (resp., $g w$-closed sets, $g w$-open sets) in $X$ will be denoted by $W C(X)$ (resp., $G W C(X), G W O(X))$. We set $g W(x)=\{U \in G W O(X): x \in U\}$. And $A$ is called a generalized $w$-open set (simply, gw-open set) if $X-A$ is $g w$ closed. Then $A$ is $g w$-open if and only if $F \subseteq w I(A)$ whenever $F \subseteq A$ and $F$ is $w$-closed. For a subset $A$ of $X, g w$-closure of $A$ and gw-interior [14] of $A$ are defined as the following:

(1) $g w C(A)=\cap\{F: A \subseteq F, F$ is $g w$-closed $\}$.

(2) $g w I(A)=\cup\{U: U \subseteq A, U$ is $g w$-open $\}$.

Theorem 2.2 ([14]). Let $\left(X, w_{X}\right)$ be a $w$-space and $A \subseteq X$.

(1) If $A$ is gw-open (gw-closed), then $g w I(A)=A(g w C(A)=A)$.

(2) If $A \subseteq B$, then $g w I(A) \subseteq g w I(B) ; g w C(A) \subseteq g w C(B)$.

(3) $g w C(X-A)=X-g w I(A) ; g w I(X-A)=X-g w C(A)$.

(4) $x \in g w I(A)$ iff there exists a gw-open set $U$ containing $x$ such that $U \subseteq A$.

(5) $x \in g w C(A)$ iff $A \cap V \neq \emptyset$ for all gw-open set $V$ containing $x$.

\section{Main Results}

Definition 3.1. Let $f: X \rightarrow Y$ be a function in $w$-spaces. Then $f$ is said to be

(1) $g W$-continuous if for $x \in X$ and for each $w$-open set $V$ containing $f(x)$, there is a $g w$-open set $U$ containing $x$ such that $f(U) \subseteq V$;

(2) $g W^{*}$-continuous if for every $w$-open set $V$ in $Y, f^{-1}(V)$ is a $g w$-open set in $X$.

Obviously we obtain the following theorem:

Theorem 3.2. Every $g W^{*}$-continuous function is $g W$-continuous.

The following example supports that the converse of the above theorem is not true in general. 
Example 3.3. Let $X=\{a, b, c, d\}$ and a $w$-structure $w=\{\emptyset,\{a, c\},\{a\},\{b\}$, $\{c\},\{a, d\}, X\}$ in $X$. Then for the power set $P(X)$ of $X, G W O(X)=P(X)-$ $\{\{b, c, d\},\{b, d\}\}$ is the set of all $g w$-open sets. Consider a function $f:(X, w) \rightarrow$ $(X, w)$ defined by $f(a)=b ; f(b)=a ; f(c)=d ; f(d)=c$. Then $f$ is $g W$ continuous. For a $w$-open set $\{a, c\}, f^{-1}(\{a, c\})=\{b, d\}$ is not gw-open, and so $f$ is not $g W^{*}$-continuous.

We recall that: Let $f: X \rightarrow Y$ be a function on $w$-spaces. Then $f$ is said to be

(1) $W$-continuous [12] if for $x \in X$ and for each $w$-open set $V$ containing $f(x)$, there is a $w$-open set $U$ containing $x$ such that $f(U) \subseteq V$;

(2) $W^{*}$-continuous [12] if for every $w$-open set $V$ in $Y, f^{-1}(V)$ is a $w$-open set in $X$.

Obviously, the following things are obtained:

Theorem 3.4. (1) Every $W$-continuous function is $g W$-continuous.

(2) Every $W^{*}$-continuous function is $g W^{*}$-continuous.

Proof. Since every $w$-open set is $g w$-open, the things are obvious.

The following example supports that the converses of the above theorem are not true in general.

Example 3.5. In Example 3.3: (1) For $d \in X$ and for a $w$-open set $V=\{b\}$ containing $f(d)$, there is no any $w$-open set $U$ containing $d$ such that $f(U) \subseteq V$. So, the $g W$-continuous function $f$ is not $W$-continuous.

(2) Consider a function $g:(X, w) \rightarrow(X, w)$ defined by $g(a)=b ; g(b)=$ $c ; g(c)=a ; g(d)=d$. Then $g$ is $g W^{*}$-continuous but not $W^{*}$-continuous.

Definition 3.6. Let $f:\left(X, w_{\tau}\right) \rightarrow\left(Y, w_{\mu}\right)$ be a function in two associated $w$-spaces with $\tau$ and $\mu$. Then $f$ is said to be

(1) $g W$-irresolute if for $x \in X$ and for each $g w$-open set $V$ containing $f(x)$, there is $g w$-open set $U$ containing $x$ such that $f(U) \subseteq V$;

(2) $g W^{*}$-irresolute if for every $g w$-open set $V$ in $Y, f^{-1}(V)$ is $g w$-open in $X$.

Theorem 3.7. (1) Every $g W^{*}$-irresolute function is $g W$-irresolute.

(2) Every $g W$-irresolute is $g W$-continuous.

(3) Every $g W^{*}$-irresolute function is $g W^{*}$-continuous.

Proof. (1) Obvious.

(2) and (3) Since every $w$-open set is gw-open, they are obtained. 
The following example supports that the converses of the above theorem are not true in general.

Example 3.8. 1) In Example 3.3, (i) consider a function $f:(X, w) \rightarrow$ $(X, w)$ defined by $f(a)=a ; f(b)=f(c)=f(d)=b$. Then $f$ is $g W$-irresolute but not $g W^{*}$-irresolute;

(ii) consider the function $g:(X, w) \rightarrow(X, w)$ defined by $g(a)=b ; g(b)=$ $c ; g(c)=a ; g(d)=d$. Then $g$ is $g W^{*}$-continuous but not $g W^{*}$-irresolute.

2) Let $X=\{a, b, c, d\}$ and $w_{X}=\{\emptyset,\{a\},\{a, b\},\{a, c\},\{a, d\},\{a, b, d\}, X\}$ be a $w$-structure in $X$. Note that:

$$
\begin{gathered}
W C(X)=\{\emptyset,\{b, c, d\},\{c, d\},\{b, d\},\{b, c\},\{c\}, X\} ; \\
G W C(X)=\{\emptyset,\{b\},\{c\},\{d\},\{b, c\},\{b, d\},\{c, d\},\{a, b, c\}, \\
\{a, c, d\},\{b, c, d\}, X\} ; \\
G W O(X)=\{\emptyset,\{a\},\{b\},\{d\},\{a, d\},\{a, c\},\{a, b\},\{a, b, c\}, \\
\{a, c, d\},\{a, b, d\}, X\} .
\end{gathered}
$$

Consider a function $h:(X, w) \rightarrow(X, w)$ defined by $h(a)=a ; h(b)=$ $c ; h(c)=b ; h(d)=d$. Then $h$ is $g W$-continuous. But for a $g w$-open set $\{b\}$, $h^{-1}(\{b\})=\{c\}$ is not $g w$-open, so $h$ is not $g W$-irresolute.

Remark 3.9. For a function from a $w$-space to a $w$-space, we have the following diagram:

$$
\begin{array}{ccc}
W^{*} \text {-continuity } & \Rightarrow & W \text {-continuity } \\
\Downarrow & & \Downarrow \\
g W^{*} \text {-continuity } & \Rightarrow & g W \text {-continuity } \\
\Uparrow & & \Uparrow \\
g W^{*} \text {-irresolute } & \Rightarrow & g W \text {-irresolute }
\end{array}
$$

Theorem 3.10. Let $f: X \rightarrow Y$ be a function in $w$-spaces. Then $f$ is $g W^{*}$-continuous if and only if for every $w$-closed set $F$ in $Y, f^{-1}(F)$ is $g w$-closed in $X$.

Proof. It is obvious.

Theorem 3.11. Let $f: X \rightarrow Y$ be a function in $w$-spaces. Then the following statements are equivalent:

(1) $f$ is $g W$-continuous.

(2) $f(g w C(A)) \subseteq w C(f(A))$ for $A \subseteq X$.

(3) $g w C\left(f^{-1}(V)\right) \subseteq f^{-1}(w C(V))$ for $V \subseteq Y$.

(4) $f^{-1}(w I(V)) \subseteq g w I\left(f^{-1}(V)\right)$ for $V \subseteq Y$ 
Proof. $(1) \Rightarrow(2)$ Let $x \in g w C(A)$. Suppose that $f(x)$ is not in $w C(f(A))$; then there exists a $w$-open set $V$ containing $f(x)$ such that $V \cap f(A)=\emptyset$. Вy $g W$-continuity, there is a $g w$-open set $U$ containing $x$ such that $f(U) \subseteq V$, and so $f(U) \cap f(A)=\emptyset$. Hence $U \cap A=\emptyset$, which is a contradiction to $x \in g w C(A)$. So, $f(g w C(A)) \subseteq w C(f(A))$.

$(2) \Rightarrow(3)$ Let $A=f^{-1}(B)$ for $B \subseteq Y$. By hypothesis, $f(g w C(A)) \subseteq$ $w C(f(A))=w C\left(f\left(f^{-1}(B)\right)\right) \subseteq w C(B)$. Hence, $g w C\left(f^{-1}(B)\right) \subseteq f^{-1}(w C(B))$.

$(3) \Rightarrow(4)$ By Theorem 2.1 and Theorem 2.2, it is obvious.

$(4) \Rightarrow(1)$ Let $V$ be any $w$-open set containing $f(x)$ for each $x \in X$. Then by hypothesis, $x \in f^{-1}(w I(V)) \subseteq g w I\left(f^{-1}(V)\right)$. So, there exists a $g w$-open set $U$ such that $x \in U \subseteq g w I\left(f^{-1}(V)\right) \subseteq f^{-1}(V)$. It implies that $f$ is $g W$ continuous.

Corollary 3.12. Let $f: X \rightarrow Y$ be a function on $w$-spaces. Then the following statements are equivalent:

(1) $f$ is $g W$-continuous.

(2) $f^{-1}(V)=g w I\left(f^{-1}(V)\right)$ for every $w$-open set $V \in Y$.

(3) $f^{-1}(B)=g w C\left(f^{-1}(B)\right)$ for every $w$-closed set $B \subseteq Y$.

Proof. From (1) of Theorem 2.2, it is obvious.

Theorem 3.13. Let $f:\left(X, w_{\tau}\right) \rightarrow\left(Y, w_{\mu}\right)$ be a function in two associated $w$-spaces with $\tau$ and $\mu$. Then the following statements are equivalent:

(1) $f$ is $g W$-irresolute.

(2) $f(g w C(A)) \subseteq g w C(f(A))$ for $A \subseteq X$.

(3) $g w C\left(f^{-1}(V)\right) \subseteq f^{-1}(g w C(V))$ for $V \subseteq Y$.

(4) $f^{-1}(g w I(V)) \subseteq g w I\left(f^{-1}(V)\right)$ for $V \subseteq Y$

Proof. Since the family of all $g w$-open sets is a weak structure in $X$, it is similar to the proof of Theorem 3.11.

Let $(X, w)$ be a $w$-space. Let $g W(x)$ (resp., $W(x))$ denote the set of all $g w$-open (resp., $w$-open) set containing $x$ in $X$. A collection $\mathcal{H}$ of subsets of $X$ is called an $m$-family [10] on $X$ if $\cap \mathcal{H} \neq \emptyset$. Let $\mathcal{H}$ be an $m$-family on $X$. Then we say that an $m$-family $\mathcal{H}$ gw-converges (resp., $w$-converges) to $x \in X$ if $\mathcal{H}$ is finer than $g W(x)$ (resp., $W(x))$ i.e., $g W(x) \subseteq \mathcal{H}$ (resp., $W(x) \subseteq \mathcal{H}$ ). Let $f: X \rightarrow Y$ be a function; then it is obvious $f(\mathcal{H})=\{f(F): F \in \mathcal{H}\}$ is an $m$-family on $Y$. 
Theorem 3.14. Let $f: X \rightarrow Y$ be a function in $w$-spaces. If $f$ is $g W$-continuous, then for an $m$-family $\mathcal{H} g w$-converging to $x \in X$, an $m$-family $<f(\mathcal{H})>=\{F: H \subseteq F$ for $H \in f(\mathcal{H})\}$ w-converges to $f(x)$.

Proof. Let $f$ be $g W$-continuous and let $\mathcal{H}$ be an $m$-family $g w$-converging to $x \in X$. By $g W$-continuity, for a $w$-open set $V$ containing $f(x)$, there exists a $g w$-open set $U$ containing $x$ such that $f(U) \subseteq V$. Since $f(g W(x)) \subseteq f(\mathcal{H})$, $V \in\langle f(\mathcal{H})>$, and so $W(f(x)) \subseteq<f(\mathcal{H})>$. Hence the $m$-family $\langle f(\mathcal{H})\rangle$ $w$-converges to $f(x)$.

Corollary 3.15. Let $f: X \rightarrow Y$ be a function on $w$-spaces. Then if $f$ is $g W^{*}$-continuous, then for an $m$-family $\mathcal{H} g w$-converging to $x \in X$, the $m$-family $<f(\mathcal{H})>$ w-converges to $f(x)$.

Proof. Since every $g W^{*}$-continuous function is $g W$-continuous, it is obtained obviously.

Corollary 3.16. Let $f: X \rightarrow Y$ be a function on $w$-spaces. Then if $f$ is $W^{*}$-continuous, then for an $m$-family $\mathcal{H} w$-converging to $x \in X$, the $m$-family $<f(\mathcal{H})>$ w-converges to $f(x)$.

Proof. Since every $W^{*}$-continuous function is $g W^{*}$-continuous, from the above corollary, it is obtained obviously.

Theorem 3.17. Let $f: X \rightarrow Y$ be a bijective function on $w$-spaces. Then $f$ is $g W^{*}$-continuous iff for an $m$-family $\mathcal{H} g w$-converging to $x \in X$, the $m$-family $f(\mathcal{H}) w$-converges to $f(x)$.

Proof. Suppose $f$ is $g W^{*}$-continuous and $\mathcal{H}$ is an $m$-family $g w$-converging to $x \in X$. By hypothesis and surjectivity, $W(f(x)) \subseteq f(g W(x)) \subseteq f(\mathcal{H})$, and so the $m$-family $f(\mathcal{H}) w$-converges to $f(x)$.

For the converse, let $U \in W(f(x))$ for $U \subseteq Y$. Since the family $g W(x)$ clearly $g w$-converges to $x$, by hypothesis, we get $W(f(x)) \subseteq f(g W(x))$ for $x \in X$. Since $f$ is injectivity, $f^{-1}(U) \in g W(x)$.

Corollary 3.18. Let $f: X \rightarrow Y$ be a bijective function on $w$-spaces. Then $f$ is $W^{*}$-continuous iff for an $m$-family $\mathcal{H} w$-converging to $x \in X$, the $m$-family $f(\mathcal{H}) w$-converges to $f(x)$. 


\section{Acknowledgments}

This study was supported by 2016 Research Grant from Kangwon National University (No. D1000625-01-01).

\section{References}

[1] P. Bhattacharyya and B. K. Lahiri; Semi-generalized closed sets in topology, Indian J. Math., 29(3) (1987), 375-382.

[2] Á. Csázár; Generalized Topology, Generalized Continuity, Acta Math. Hungar., 96 (2002), 351-357.

[3] J. Dontchev and H. Maki; On sg-closed sets and semi- $\lambda$-closed sets, Q \& A in General Topology, 15(1997), 259-266.

[4] D. C. Kent and W. K. Min; Neighborhood Spaces, International Journal of Mathematics and Mathematical Sciences, 32(7) (2002), 387-399.

[5] N. Levine; Generalized closed sets in topology, Rend. Cir. Mat. Palermo, 19(1970), 89-96.

[6] N. Levine; Semi-open sets and semi-continuity in topological spaces, Ams. Math. Monthly, 70(1963), 36-41.

[7] H. Maki, J. Umehara and T. Noiri; Every topological space is pre- $T_{\frac{1}{2}}$, Mem. Fac. Sci. Kochi Univ. Ser. A, 17(1996), 33-42.

[8] H. Maki; On generalizing semi-open and preopen sets, Report for Meeting on Topological Spaces Theory and its Applications, August 1996, Yatsushiro College of Technology, 1318.

[9] A. S. Mashhour, M. E. Abd El-Monsef and S. N. El-Deeb; On precontinuous and weak precontinuous mappings, Proc. Math. and Phys. Soc. Egypt, 53 (1982), 47-53.

[10] W. K. Min; Some Results on Generalized Topological Spaces and Generalized Systems, Acta Math. Hungar., 108 (1-2) (2005), 171-181.

[11] W. K. Min; On Weak Neighborhood Systems and Spaces, Acta Math. Hungar., 121(3) (2008), 283-292.

[12] W. K. Min and Y. K. Kim; On Weak Structures and $w$-spaces, Far East Journal of Mathematical Sciences, 97(5) (2015), 549-561.

[13] W. K. Min and Y. K. Kim; On Product Weak Structures and Product w-Spaces, International Journal of Mathematical Analysis, 9(35) (2015), 1715-1721.

[14] W. K. Min and Y. K. Kim; On generalized $w$-closed sets in $w$-spaces, International Journal of Pure and Applied Mathematics, 110(2) (2016), 327-335.

[15] O. Njastad; On some classes of nearly open sets, Pacific Journal of Mathematics, 15(3)(1964), 961-970.

[16] F. Siwiec; On Defining a Space by a Weak Base, Pacific Journal of Mathematics, 52(1) (1974), 351-357. 